\title{
Sternal wound infection after coronary artery bypass graft surgery: Validation of existing risk scores
}

\author{
Mical Paul, MD, a,b Aeyal Raz, MD, PhD, ${ }^{\mathrm{c}}$ Leonard Leibovici, MD, ${ }^{\mathrm{a}}$ Hefziba Madar, RN, ${ }^{\mathrm{b}}$ Rita Holinger, RN, ${ }^{\mathrm{b}}$ and \\ Bina Rubinovitch, $\mathrm{MD}^{\mathrm{b}}$
}

Earn CME credits at http:// cme.ctsnetjournals.org
From the Department of Internal Medicine E, ${ }^{\text {a }}$ Rabin Medical Center, Beilinson Hospital and Sackler Faculty of Medicine, TelAviv University, Tel-Aviv, Israel; the Infection Control Unit, ${ }^{\text {b }}$ Rabin Medical Center, Beilinson Hospital, Tel-Aviv, Israel; and the Department of Anesthesia, Rabin Medical Center, Beilinson Hospital, Tel-Aviv, Israel.

Received for publication July 30, 2006; revisions received Sept 30, 2006; accepted for publication Oct 9, 2006.

Address for reprints: Mical Paul, MD, Internal Medicine $\mathrm{E}$ and Infectious Diseases Unit, Rabin Medical Center, Beilinson Hospital, Petah Tikva, 49100, Israel (E-mail: pillpel@zahav.net.il).

J Thorac Cardiovasc Surg 2007;133:397-403 $0022-5223 / \$ 32.00$

Copyright () 2007 by The American Association for Thoracic Surgery

doi:10.1016/j.jtcvs.2006.10.012
Objective: Prediction of surgical site infection and mortality after cardiac surgery might allow for interventions to reduce adverse outcomes. We sought to evaluate existing risk scores.

Methods: We included 809 consecutive patients undergoing coronary artery bypass surgery. Data were collected prospectively. Infections were defined as deep sternal wound infection or mediastinitis by using established criteria and evaluated 60 days after surgical intervention. All-cause mortality was assessed at 30 days and 6 months. We assessed the ability of the National Nosocomial Infections Surveillance risk index, the EuroSCORE, and the Society of Thoracic Surgeons risk score to predict infection and mortality. Discrimination was assessed using the area under the receiver operating curve.

Results: The rate of surgical site infection was 3.6\% (29/809 patients). The National Nosocomial Infections Surveillance risk index showed moderate discrimination for infection (area under the receiver operating curve of 0.64 ) and poor ability to stratify patients into infection risk groups. The EuroSCORE predicted infection and 30-day and 6-month mortality with good discrimination (area under the receiver operating curve of $0.72,0.78$, and 0.77 , respectively). Ranking patients by the EuroSCORE and dividing the cohort into 3 roughly equal risk groups yielded an ascending risk for infection of $0.7 \%, 3.0 \%$, and $7.2 \%$. The preoperative and intraoperative Society of Thoracic Surgeons risk scores showed good discrimination for surgical site infection (area under the receiver operating curve of 0.72 and 0.76 , respectively) and excellent discrimination for early and late mortality (area under the receiver operating curve of $>0.80$ ). Risk grouping based on the Society of Thoracic Surgeons score yielded an ascending risk for infection of $0.7 \%, 3.6 \%$, and $6.4 \%$.

Conclusions: The EuroSCORE and the Society of Thoracic Surgeons risk score can be used for joint risk stratification for surgical site infection and mortality. Both scores performed better than the National Nosocomial Infections Surveillance risk index.

$\mathrm{S}$ urgical site infection (SSI) is an infrequent but devastating complication after cardiac surgery. Deep sternal wound infections and mediastinitis are associated with increased mortality, repeated surgical procedures, prolonged hospital stay, and increased costs. ${ }^{1-3}$

Several risk scores for SSI have been developed. The National Nosocomial Infections Surveillance (NNIS) risk index is well accepted for general surgery. ${ }^{4}$ However, it was not adapted specifically for coronary artery bypass graft (CABG) operations. This score is based on the American Society of Anesthesiologists (ASA) score, wound class, and operation duration. It does not take into account particular risk factors among patients undergoing $\mathrm{CABG}$ and permits little variability because CABG operations are always clean and patients' ASA scores are greater than 2 per 


\section{Abbreviations and Acronyms}

ASA = American Society of Anesthesiologists

$\mathrm{CABG}=$ coronary artery bypass grafting

NNIS $=$ National Nosocomial Infections Surveillance

$\mathrm{ROC}=$ receiver operating characteristic

SSI = surgical site infection

STS $=$ Society of Thoracic Surgeons definition. Fowler and colleagues ${ }^{2}$ developed a simple bedside risk score using the Society of Thoracic Surgeons (STS) National Cardiac Database, including more than 300,000 patients undergoing CABG surgery. The STS score predicts the risk for major infections, including SSIs or septicemia, after CABG. A preoperative score, including variables available before surgical intervention and an intraoperative score, including preoperative and intraoperative variables, performed well in the validation sample of the STS database. Finally, the EuroSCORE is a risk score for 30-day mortality after cardiac surgery that has been well validated worldwide. ${ }^{5-8}$ It has been proposed that the EuroSCORE can predict other adverse outcomes, including infections, after surgical intervention. ${ }^{9}$ The similarity between risk factors for SSI and mortality might permit joint risk stratification.

We validated these 3 risk scores in a sample of 809 patients undergoing $\mathrm{CABG}$ during 1 year in a single center in Israel. Our objective was to compare the performance of these risk scores and to validate the STS scores of patients outside the STS database.

\section{Materials and Methods}

The study was performed at the Department of Cardiothoracic Surgery at Rabin Medical Center, Beilinson Hospital, a primaryand tertiary-care university-affiliated hospital. The hospital belongs to the largest health maintenance organization in Israel (Clalit Health Services), which insures about $60 \%$ of its population (more than 3.7 million members of various ethnic origin). The department of thoracic surgery conducts the largest number of cardiothoracic operations in Israel, including CABG, valve, and lung operations and cardiac transplantation. A prospective surveillance was performed throughout 2004 (August omitted) by the infection control team. Included were all consecutive patients undergoing $\mathrm{CABG}$ with or without an additional operation. A dedicated infection-control nurse visited the department daily and collected data by using a standardized case report form. The antibiotic prophylaxis recommended at the time of the study was vancomycin for valve surgery and cefazolin for all other operations, both administered as $1 \mathrm{~g}$ within 60 minutes before incision. Repeat administration was recommended for cefazolin after disconnection from bypass or after 4 hours. No prophylaxis was recommended after surgical intervention.

Preoperative data collected included patient demographics and detailed data regarding hospital stay and invasive procedures be- fore surgical intervention, preoperative risk factors using EuroSCORE definitions, ${ }^{10}$ hemoglobin A1c (HBAlc) when available, previous antibiotic use, and preoperative patient preparation. Intraoperative data included the type of operation and procedures performed; operation duration; bypass use and duration; hourly arterial glucose level, continuous temperature for on-pump surgery, and mean intraoperative temperature for off-pump surgery; timing, dosing, and type of antibiotic prophylaxis; administration of blood products; and mean fraction of inspired oxygen and $\mathrm{PaO}_{2}$. Postoperative data included temperature and oxygenation on arrival to the intensive care unit; duration of hypothermia, mechanical ventilation, and intensive care unit stay; complications during the first 3 postoperative days; and 30-day and 6-month all-cause mortality. The EuroSCORE and NNIS risk index values were collected prospectively. SSIs were diagnosed and classified according to Centers for Disease Control and Prevention criteria. ${ }^{11}$

Data were obtained from patients' hospital charts and electronic databases. The electronic databases available for routine work at our hospital present data from all hospitals, primary care clinics, and laboratories belonging to the health maintenance organization. Thus data on background conditions, previous hospitalizations, HBA1c, and postdischarge follow-up with hospital or clinic notes, microbiologic studies, and surgical procedures were available. Data for this study were collected as part of the routine surveillance conducted by the infection control unit at our hospital. The study was conducted in accordance with the requirements of the local ethics committee.

The primary outcome assessed was SSI 60 days after surgical intervention, including deep sternal wound infection or mediastinitis. Because most continuous variables did not have a normal distribution, the Mann-Whitney $U$ test (nonparametric) was used for comparisons. For comparisons of dichotomous data, we used the $\chi^{2}$ test. A multiple logistic regression model was constructed to assess independent risk factors for SSI. Variables associated with SSI at a $P$ value of less than .1 on univariate analysis were entered by using stepwise forward logistic regression. We did not include composite risk scores in the model because we assessed their individual components.

We assessed the ability of each of the risk scores to predict occurrence of the primary outcome and mortality. We used the area under the receiver operating characteristic (ROC) curve with 95\% confidence intervals to assess the discriminatory ability of each of the risk scores. Calibration was assessed by using the Hosmer-Lemeshow goodness-of-fit statistic. For each score, we grouped patients into low-, intermediate-, and high-risk patients to assess the ability of the score to identify a clinically meaningful percentage of the study population at high risk for infection. All analyses were conducted with SPSS 13.0 statistical software (SPSS, Inc).

\section{Results}

We evaluated 809 consecutive patients undergoing CABG throughout the study period. An operation in addition to CABG was performed in $121(15 \%)$ patients, most commonly valve surgery (102 patients). Patient characteristics are detailed in Table 1. Compared with the STS cohort, diabetes mellitus was more prevalent in our cohort (phar- 
macologically treated diabetes in 330 patients, 41\%). The average body mass index was lower in our cohort, whereas other risk factors, including age, ejection fraction, and repeated surgical intervention, were similar. Chronic lung disease was less frequent but was defined differently. More operations were performed as emergency operations in our cohort (89/809 [11\%]), whereas other operative characteristics, including operation duration and valve and on-pump operations, were similar. Mortality at 30 days was $4.8 \%$ (39/809).

The overall rate of SSI was 3.6\% (29/809 patients). Ten (34\%) infections were diagnosed after discharge, and 3 (10\%) were diagnosed more than 30 days after surgical intervention. Invasive interventions were required for 20 $(69 \%)$ patients. The average length of stay in the hospital for patients with SSIs was $27.9 \pm 19.9$ days compared with $7.3 \pm 7.4$ days for patients without SSIs $(P<.001)$. Six-month all-cause mortality rates were $34.5 \%(10 / 29)$ versus $7.6 \%$ (59/780), respectively $(P<.001)$. Significant risk factors for SSI on univariate analysis are detailed in Table 1. Perioperative temperature and oxygenation levels were not associated with SSI. Off-pump surgery and isolated $\mathrm{CABG}$ were associated with a lower rate of SSI. Compared with patients undergoing on-pump operations, off-pump patients had significantly less combined $(18 \%$ [114/633] vs 3.4\% [6/174], $P>.001)$ and emergency (12.4\% [78/631] vs 5.2\% [9/174], $P=.007)$ operations. Vancomycin prophylaxis was used in $84.2 \%(96 / 114)$ of combined operations versus $4.5 \%$ (29/639) of isolated CABG operations. On multivariate analysis, chronic obstructive pulmonary disease, an operation in addition to $\mathrm{CABG}$, and diabetes mellitus remained significantly associated with SSI (Table 2).

\section{NNIS Risk Index}

We used the ASA score as recorded by the anesthesiologists preoperatively (data missing for 108 patients) and an operation duration cutoff of 240 minutes, as defined by the NNIS system for "chest-only" SSIs. ${ }^{4}$ Nearly all patients were classified as risk index 1 or 2 , as expected. The respective SSI rates for these risk groups were $2.0 \%$ and $5.8 \%$ (Table 3). The discrimination of the score for SSI and mortality was moderate (Table 4).

\section{EuroSCORE}

The EuroSCORE was collected prospectively, adhering to original variable definitions. The mean additive and logistic EuroSCORE values in our cohort were 5.18 ( \pm 3.3 ) and 7.0 $( \pm 9.0)$, respectively, which is higher than values reported from European cohorts (range, 3.1-4.7). ${ }^{12}$

Ranking patients by the EuroSCORE and dividing the cohort into 3 roughly equal groups yielded a high-risk group consisting of $33 \%$ of patients with an observed rate of SSI of $7.2 \%$ (Table 3). The discriminative power of the EuroSCORE with regard to prediction of SSI was good (areas under the ROC curve of 0.72 for the additive model and 0.73 for the logistic score). Originally modeled to predict mortality, the EuroSCORE performed well in our cohort, with an area under the ROC curve of 0.78 for 30 -day mortality and 0.77 for 6-month mortality (Table 4).

\section{STS Risk Score}

Several variables in our database were defined differently from the original variable definitions used in the STS database. We defined chronic lung disease and diabetes as conditions necessitating chronic treatment; myocardial infarction was recorded within 3 months before the operation. We did not collect data on insertion of an intra-aortic balloon pump.

We ranked our cohort by the STS risk score and divided it into 3 risk groups (Table 3). The number of patients within each risk group was similar to the distribution observed in the original STS cohort (low risk, 35\% vs 31\% of patients; intermediate risk, $30 \%$ vs 30\%; high risk, $35 \%$ vs $38 \%$ for our cohort versus the STS database; data supplied by the authors). The observed rate of infections in the high-risk group was $6.4 \%$ (mean predicted value for the group, 5.3\%). Both the preoperative and intraoperative (omitting intra-aortic balloon pump) scores discriminated well between patients with and without SSIs (Table 4). The area under the ROC curve was 0.72 for the preoperative score and 0.76 for the intraoperative score, indicating good discrimination. The respective values in the original STS validation cohort were 0.70 and 0.71 . Calibration was assessed against the predicted probability of infection, as reported in the original model derivation. The HosmerLemeshow $\chi^{2}$ value was $9.86(P>.25)$ for the preoperative and $6.55(P>.5)$ for the intraoperative scores, indicating adequate calibration. The score we used was designed to predict major infections but predicted mortality in our cohort with excellent discrimination (areas under the ROC curves of $\geq 0.80$ for both the preoperative and intraoperative models, Table 4).

\section{Discussion}

We assessed the ability of several risk indexes to predict deep sternal wound infections in a prospectively collected cohort of patients undergoing CABG. Our study shows good discriminative power and adequate calibration for the STS preoperative and intraoperative risk scores. The EuroSCORE, originally designed to predict mortality, predicted SSI with good discrimination. We found that both the STS score and the EuroSCORE were highly predictive for short- and long-term survival after surgical intervention. Performance of the NNIS risk index for prediction of SSI was inferior to that of the STS risk score and the EuroSCORE.

The STS risk score was developed by using a national database assessing mainly cardiovascular process and out- 
TABLE 1. Characteristics of patients with and without SSIs*

\begin{tabular}{|c|c|c|c|}
\hline Variable & No SSI (780 patients) & SSI (29 patients) & $P$ value \\
\hline \multicolumn{4}{|l|}{ Demography } \\
\hline \multirow[t]{2}{*}{ Age } & $65.5( \pm 11.0)$ & $69.43( \pm 10.1)$ & .05 \\
\hline & $66.5(31.1-91.4)$ & $72.1(46.9-83.7)$ & \\
\hline Female sex & $165(21.2 \%)$ & $9(31.0 \%)$ & .204 \\
\hline Elective operationt & $236(30.4 \%)$ & $13(44.8 \%)$ & .098 \\
\hline \multirow[t]{2}{*}{ Hospital stay before operation $\ddagger$} & $7 d( \pm 7)$ & $7 d( \pm 7)$ & .381 \\
\hline & $3 d(0-50)$ & $2 d(0-27)$ & \\
\hline \multicolumn{4}{|l|}{ Preoperative risk factors } \\
\hline \multirow[t]{2}{*}{ BMI§ } & $27.9( \pm 4.8)$ & $28.0( \pm 4.6)$ & .898 \\
\hline & $27.4(17.2-55.2)$ & $26.8(21.8-39.0)$ & \\
\hline Smoker within $30 \mathrm{~d}$ of operation & $251(33.7 \%)$ & $7(25.9 \%)$ & .398 \\
\hline COPD & $43(5.5 \%)$ & $5(17.2 \%)$ & .009 \\
\hline Diabetes & & & .004 \\
\hline Oral treatment & $231(30.0 \%)$ & $13(44.8 \%)$ & \\
\hline Insulin treatment & $79(10.3 \%)$ & $7(24.1 \%)$ & \\
\hline Chronic renal failure (creatinine $>2.2 \mathrm{mg} / \mathrm{dL}$ ) & $18(2.3 \%)$ & $1(3.4 \%)$ & 691 \\
\hline Moderate-severe left ventricular dysfunction & $234(30.0 \%)$ & $11(37.9 \%)$ & .361 \\
\hline \multicolumn{4}{|l|}{ Operative information } \\
\hline Adequate antibiotic prophylaxis $\|$ & $414(54.9 \%)$ & $12(42.9 \%)$ & .209 \\
\hline Administration of prophylaxis & $750(96.2 \%)$ & $25(86.2 \%)$ & .029 \\
\hline Adequate timing before operation & $481(65.7 \%)$ & $12(52.2 \%)$ & .179 \\
\hline Recommended agent used & $713(93.0 \%)$ & $24(82.8 \%)$ & .040 \\
\hline Adequate repeat dose & $675(90.1 \%)$ & $24(96.0 \%)$ & .500 \\
\hline Discontinuation after operation & $770(99.2 \%)$ & $28(100 \%)$ & 1.000 \\
\hline Emergency operation & $84(10.8 \%)$ & $5(17.9 \%)$ & .240 \\
\hline Repeated CABG & $40(5.1 \%)$ & $3(10.3 \%)$ & .220 \\
\hline Operation in addition to $\mathrm{CABG}$ & $109(14.0 \%)$ & $12(41.4 \%)$ & $<.001$ \\
\hline Off-pump operation & $174(22.3 \%)$ & 0 & .005 \\
\hline Double thoracic use & $576(73.8 \%)$ & $22(75.9 \%)$ & .808 \\
\hline Blood product administration & $448(61.2 \%)$ & $23(88.5 \%)$ & .005 \\
\hline \multirow[t]{2}{*}{ Operation duration (min) } & $229.5( \pm 83.7)$ & $266.9( \pm 59.6)$ & .001 \\
\hline & $215(60-1074)$ & $260(178-461)$ & \\
\hline \multicolumn{4}{|l|}{ Glucose control } \\
\hline \multirow[t]{2}{*}{ HBA1c (\%)\# } & $8.0( \pm 1.8)$ & $8.2( \pm 2.0)$ & .858 \\
\hline & $7.7(4.9-12.8)$ & $7.4(6.5-12.0)$ & \\
\hline \multirow[t]{2}{*}{ Preoperative measurement } & $140( \pm 64)$ & $166( \pm 61)$ & .017 \\
\hline & $119(42-548)$ & $143(88-279)$ & \\
\hline \multirow[t]{2}{*}{ Mean intraoperative } & $151( \pm 38)$ & $171( \pm 41)$ & .003 \\
\hline & $143(73-357)$ & $164(96-188)$ & \\
\hline \multirow[t]{2}{*}{ Mean day 1 postoperatively } & $185( \pm 35)$ & $190( \pm 39)$ & .452 \\
\hline & $179(109-356)$ & $187(126-316)$ & \\
\hline \multirow[t]{2}{*}{ Mean day 2 postoperatively } & $153( \pm 32)$ & $161( \pm 32)$ & .227 \\
\hline & $149(73-327)$ & $154(111-244)$ & \\
\hline \multicolumn{4}{|l|}{ Postoperative course } \\
\hline \multirow[t]{2}{*}{ Duration of mechanical ventilation (h:min) } & $12: 02(4: 32)$ & $12: 04(3: 53)$ & .760 \\
\hline & $11: 45(0-23: 40)$ & $12: 30(3: 00-19: 30)$ & \\
\hline Reopening & $32(4.2 \%)$ & $1(4.3 \%)$ & .975 \\
\hline Infectious complications ${ }^{* *}$ & $29(3.8 \%)$ & $5(21.7 \%)$ & $<.001$ \\
\hline Other complications ${ }^{* *}$ & $27(3.7 \%)$ & $1(4.8 \%)$ & .801 \\
\hline 30-d all-cause mortality & $39(5.0 \%)$ & 0 & .217 \\
\hline 60-d all-cause mortality & $59(7.6 \%)$ & $10(34.5 \%)$ & $<.001$ \\
\hline
\end{tabular}


TABLE 1. Continued

\begin{tabular}{|c|c|c|c|}
\hline Variable & No SSI (780 patients) & SSI (29 patients) & $P$ value \\
\hline \multicolumn{4}{|l|}{ Risk scores } \\
\hline NNIS risk index & $\begin{array}{c}1.12( \pm 0.38) \\
1(0-2)\end{array}$ & $\begin{array}{c}1.17( \pm 0.39) \\
1(0-2)\end{array}$ & .478 \\
\hline Additive EuroSCORE & $\begin{array}{c}5.1( \pm 3.3) \\
5(0-18)\end{array}$ & $\begin{array}{c}7.4( \pm 2.6) \\
8(3-12)\end{array}$ & $<.001$ \\
\hline Logistic EuroSCORE & $\begin{array}{c}6.82( \pm 8.9) \\
4(1-76)\end{array}$ & $\begin{array}{c}11.1( \pm 7.89) \\
10(2-31)\end{array}$ & $<.001$ \\
\hline STS preoperative & $\begin{array}{c}8.58( \pm 4.76) \\
8(0-26)\end{array}$ & $\begin{array}{c}11.93( \pm 3.40) \\
12(5-18)\end{array}$ & $<.001$ \\
\hline STS intraoperative & $\begin{array}{c}9.82( \pm 5.35) \\
10(0-33)\end{array}$ & $\begin{array}{c}14.59( \pm 4.38) \\
15(7-25)\end{array}$ & $<.001$ \\
\hline
\end{tabular}

Values of continuous variables are given as means (standard deviations) on the first line and medians (ranges) in the second line. SSI, Surgical site infection; $B M I$, body mass index; COPD, chronic obstructive pulmonary disease; $C A B G$, coronary artery bypass grafting; HBA1c, Hemoglobin A1c; NNIS, National Nosocomial Infections Surveillance; STS, Society of Thoracic Surgeons. *Percentages might not total $100 \%$ because of missing data. $†$ Admitted for surgical intervention from home. $\$$ Among patients hospitalized before surgical intervention. §Data available for 661 patients without SSIs (85\%) and 24 patients with SSIs (83\%). \|Defined as fulfilling all of the variables listed below: administration of antibiotic prophylaxis; first dose administered within 0 to 60 minutes before incision; use of the recommended antibiotic agent; repeat dose of cefazolin after disconnection from bypass for on-pump operations or after 4 hours for off-pump operations; and discontinuation of prophylaxis after surgical intervention. q[Operation performed immediately on referral to surgeon or before the beginning of the next working day. \#Data available for patients with known diabetes, in whom HBA1c measurement was performed within 3 months before surgical intervention: $113(36 \%)$ of 310 patients without SSIs and 7 (35\%) of 20 patients with SSIs. **Complications during first 3 postoperative days. Other complications include acute myocardial infarction, cerebrovascular event, reintubation without reopening, and acute renal failure.

come measures. ${ }^{2}$ There were several differences between our methods and the STS database. Risk factors were collected by using the EuroSCORE definitions. ${ }^{10}$ The primary outcome of interest in our study included deep sternal wound infection and mediastinitis, whereas Fowler and colleagues $^{2}$ included superficial site infection and septicemia in their primary end point of major infection. Our surveillance included postdischarge follow-up for SSI, and indeed, more than $30 \%$ of infections were identified after discharge. Thus the absolute number of infections in our cohorts is not comparable. However, despite these differences, the model performed very well in our cohort. In the highest risk group, consisting of one third of the cohort, the rate of SSI was $6.4 \%$ compared with $0.7 \%$ in the low-risk group. An important risk factor for SSI emerging in our cohort was intraoperative glucose control (Tables 1 and 2), which was not assessed in the STS database. It should be investigated whether inclusion of perioperative glucose values might improve the performance of the model. Interestingly, the score predicted mortality with excellent discrimination.

The EuroSCORE is a well-validated score for prediction of mortality after cardiac surgery. ${ }^{6-8}$ All variables included in the EuroSCORE were prospectively collected by using their original definitions. The score predicted both shortterm (30-day) and long-term (6-month) mortality rates with very good discrimination in our cohort (area under the ROC curve of 0.78 and 0.77 , respectively), somewhat better than described in an earlier cohort at our center. ${ }^{5}$ Performance of the EuroSCORE with regard to prediction of SSI has been assessed in a single study previously. ${ }^{9}$ In our dataset the discriminative power of the EuroSCORE for SSI was better (area under the ROC curve of 0.72 for SSI in our cohort vs 0.57 for deep sternal wound infection ${ }^{9}$ ).

The NNIS risk index is widely used for benchmarking within and between centers. We show that this score is less

TABLE 2. Multiple logistic regression model for surgical site infection ( $\mathrm{n}=793$, Wald $\chi^{2}$ for the model $=295,1 \mathrm{df}$, $P<.0001$; Hosmer-Lemeshow $\chi^{2}=0.818,4 d f, P=.936$ )

\begin{tabular}{llcc}
\hline Variable* $^{*}$ & $\begin{array}{c}\text { Odds } \\
\text { ratio }\end{array}$ & $\begin{array}{c}\text { 95\% Confidence } \\
\text { interval }\end{array}$ & $\begin{array}{c}\boldsymbol{P} \\
\text { value }\end{array}$ \\
\hline COPD & 3.50 & $1.19-10.27$ & .023 \\
Off-pump surgery & 0 & & .995 \\
Operation in addition to CABG & 3.71 & $1.67-8.24$ & .001 \\
Diabetes mellitust & 3.14 & $1.38-7.17$ & .007
\end{tabular}

$C O P D$, Chronic obstructive pulmonary disease; $C A B G$, coronary artery bypass grafting. *Variables included in the model: age, diabetes mellitus, chronic obstructive pulmonary disease, severe congestive heart failure, elective surgical intervention, administration of antibiotic prophylaxis, offpump surgery, and blood product administration. Operation duration and glucose levels before and during the operation were correlated with an operation in addition to CABG and diabetes mellitus, respectively, and thus were not entered into the model. $†$ Replacing diabetes mellitus with preoperative and intraoperative glucose levels resulted in a final model including the same variables and mean intraoperative glucose level of greater than $140 \mathrm{mg} / \mathrm{dL}$ significantly associated with SSI (odds ratio, 3.32; 95\% confidence interval, 1.11-9.92; $P=.032$ ). 
TABLE 3. Grouping of patients by preoperative risk of infection using the different risk scores

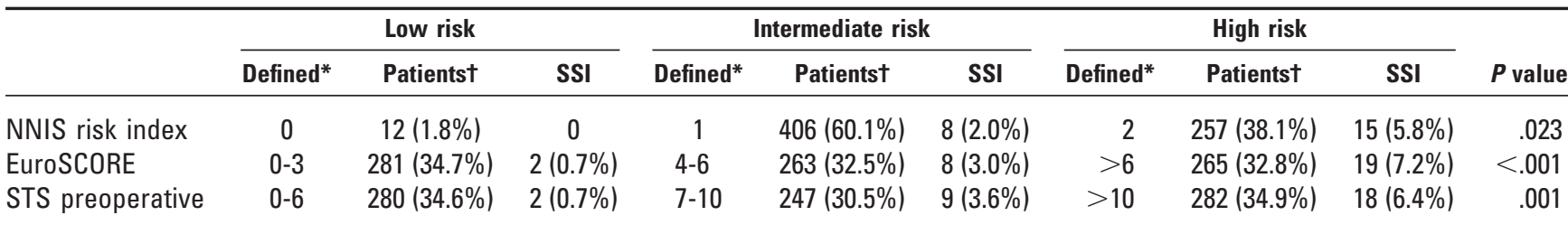

SSI, Surgical site infection; NNIS, National Nosocomial Infections Surveillance; STS, Society of Thoracic Surgeons. *Definition of the risk group according to each of the risk scores. $\dagger$ Number (percentage of all patients). All patients $(n=809)$ were evaluated for the EuroSCORE and Society of Thoracic Surgeons score; 675 patients were evaluated for the National Nosocomial Infections Surveillance risk index because of missing data (American Society of Anesthesiologists score or operation duration) for 134 patients.

TABLE 4. Discrimination of each of the risk scores assessed for deep sternal wound infection and mediastinitis (SSI) and mortality

\begin{tabular}{lccc}
\hline & SSI & 30-d all-cause mortality & 6-mo all-cause mortality \\
\hline NNIS risk index & $0.64(0.53-0.76)$ & $0.65(0.56-0.74)$ & $0.64(0.57-0.71)$ \\
EuroSCORE additive & $0.72(0.64-0.80)$ & $0.78(0.73-0.84)$ & $0.77(0.72-0.82)$ \\
EuroSCORE logistic & $0.73(0.65-0.81)$ & $0.78(0.73-0.84)$ & $0.77(0.72-0.82)$ \\
STS preoperative & $0.72(0.64-0.79)$ & $0.80(0.75-0.86)$ & $0.81(0.76-0.85)$ \\
STS intraoperative & $0.76(0.68-0.83)$ & $0.82(0.77-0.88)$ & $0.82(0.77-0.87)$
\end{tabular}

Values are presented as the area under the receiver operating characteristic curve with $95 \%$ confidence intervals. SSI, Surgical site infection; NNIS, National Nosocomial Infections Surveillance; STS, Society of Thoracic Surgeons.

well adapted for SSI after cardiac surgery than the scores specifically designed for these operations.

There are limitations and strengths to our study. We used a single-center, small cohort of patients. However, the small cohort permitted close and detailed surveillance. The electronic records used in the hospital permitted preadmission and postdischarge data collection. Thus the presence or absence of an SSI was confirmed at 60 days postoperatively in all but 2 patients, in whom no SSIs were recorded in our hospital, but postdischarge follow-up was not available. All-cause mortality was ascertained in all patients. We included only deep and organ space sternum infections in our outcome because the diagnosis of these infections is unequivocal, and these infections are associated with significant morbidity and mortality. The small number of outcomes in our study precluded the assessment of specific pathogens. Optimally, a risk score should be able to predict infections that can be prevented, such as those caused by Staphylococcus aureus. Finally, we did not assess the effect of risk stratification prospectively. Ultimately, risk stratification should be used to improve patient outcomes.

Interventions that have been shown to reduce SSIs include antibiotic prophylaxis, ${ }^{13}$ surveillance for and decontamination of Staphylococcus aureus, ${ }^{14,15}$ tight perioperative glucose control, ${ }^{16-20}$ and optimal skin preparation. ${ }^{21}$ In our study vancomycin was used mainly in combined operations, and the recommended dose of cefazolin for on-pump operations was $1 \mathrm{~g}$. Both combined and on-pump operations were associated with an increased rate of SSI. Previous studies have suggested that $\beta$-lactams might be superior to glycopeptides for the prevention of SSIs,${ }^{22}$ even in locations with a high prevalence of methicillin-resistant Staphylococcus aureus, ${ }^{23}$ and that the use of $1 \mathrm{~g}$ of cefazolin dosing might be insufficient for operations with cardiopulmonary bypass. ${ }^{24}$ Other interventions, such as management of chronic obstructive pulmonary disease before admission, should be explored. The benefit of surgical intervention versus percutaneous coronary intervention, when possible, can be reconsidered in light of preoperative assessment, ${ }^{25-27}$ as well as the necessity of concomitant surgical intervention. Although general measures for prevention of infections acquired during surgical intervention, such as antibiotic prophylaxis, apply to all patients, special interventions can be considered for patients at high risk for SSIs. The 2 scores we validated can be used to define patients at high risk, candidates for special interventions to reduce SSIs. The threshold of predicted SSI used to implement an intervention should be assessed. Intraoperative and postoperative risk stratification will allow for close monitoring and early diagnosis of infections among high-risk patients.

In summary, we show that both the STS risk score and the EuroSCORE can be used to identify SSI risk among patients undergoing CABG. The similarity of risk factors allows for joint risk stratification for SSI and mortality. Future studies should assess the effect of implementing risk assessment, with or without special interventions targeting high-risk patients. 
We thank Professor Vance G. Fowler, Jr, and colleagues ${ }^{2}$ for supplying additional data for their study.

\section{References}

1. Coskun D, Aytac J, Aydinli A, Bayer A. Mortality rate, length of stay and extra cost of sternal surgical site infections following coronary artery bypass grafting in a private medical centre in Turkey. $J$ Hosp Infect. 2005;60:176-9.

2. Fowler VG Jr, O'Brien SM, Muhlbaier LH, Corey GR, Ferguson TB, Peterson ED. Clinical predictors of major infections after cardiac surgery. Circulation. 2005;112(suppl):I358-65.

3. Toumpoulis IK, Anagnostopoulos CE, Derose JJ Jr, Swistel DG. The impact of deep sternal wound infection on long-term survival after coronary artery bypass grafting. Chest. 2005;127:464-71.

4. National Nosocomial Infections Surveillance (NNIS) System Report. Data summary from January 1992 through June 2004, issued October 2004. Am J Infect Control. 2004;32:470-85.

5. Berman M, Stamler A, Sahar G, Georghiou GP, Sharoni E, Brauner R, et al. Validation of the 2000 Bernstein-Parsonnet score versus the EuroSCORE as a prognostic tool in cardiac surgery. Ann Thorac Surg. 2006;81:537-40.

6. Nashef SA, Roques F, Michel P, Gauducheau E, Lemeshow S, Salamon R. European system for cardiac operative risk evaluation (EuroSCORE). Eur J Cardiothorac Surg. 1999;16:9-13.

7. Nashef SA, Roques F, Hammill BG, Peterson ED, Michel P, Grover FL, et al. Validation of European System for Cardiac Operative Risk Evaluation (EuroSCORE) in North American cardiac surgery. Eur J Cardiothorac Surg. 2002;22:101-5.

8. Yap CH, Reid C, Yii M, Rowland MA, Mohajeri M, Skillington PD, et al. Validation of the EuroSCORE model in Australia. Eur J Cardiothorac Surg. 2006;29:441-6.

9. Toumpoulis IK, Anagnostopoulos CE, DeRose JJ, Swistel DG. Does EuroSCORE predict length of stay and specific postoperative complications after coronary artery bypass grafting? Int J Cardiol. 2005;105: $19-25$

10. Roques F, Nashef SA, Michel P, Gauducheau E, de Vincentiis C, Baudet E, et al. Risk factors and outcome in European cardiac surgery: analysis of the EuroSCORE multinational database of 19030 patients. Eur J Cardiothorac Surg. 1999;15:816-22.

11. Garner JS, Jarvis WR, Emori TG, Horan TC, Hughes JM. CDC definitions for nosocomial infections, 1988. Am J Infect Control. 1988;16:128-40.

12. Roques F, Nashef SA, Michel P, Pinna Pintor P, David M, Baudet E, et al. Does EuroSCORE work in individual European countries? Eur J Cardiothorac Surg. 2000;18:27-30.

13. Bratzler DW, Houck PM. Antimicrobial prophylaxis for surgery: an advisory statement from the National Surgical Infection Prevention Project. Clin Infect Dis. 2004;38:1706-15.

14. Kluytmans JA, Mouton JW, VandenBergh MF, Manders MJ, Maat $\mathrm{AP}$, Wagenvoort $\mathrm{JH}$, et al. Reduction of surgical-site infections in cardiothoracic surgery by elimination of nasal carriage of Staphylococcus aureus. Infect Control Hosp Epidemiol. 1996;17:780-5.
15. Nicholson MR, Huesman LA. Controlling the usage of intranasal mupirocin does impact the rate of Staphylococcus aureus deep sternal wound infections in cardiac surgery patients. Am J Infect Control. 2006;34:44-8.

16. Carr JM, Sellke FW, Fey M, Doyle MJ, Krempin JA, de la Torre R, et al. Implementing tight glucose control after coronary artery bypass surgery. Ann Thorac Surg. 2005;80:902-9.

17. Furnary AP, Zerr KJ, Grunkemeier GL, Starr A. Continuous intravenous insulin infusion reduces the incidence of deep sternal wound infection in diabetic patients after cardiac surgical procedures. Ann Thorac Surg. 1999;67:352-60.

18. Furnary AP, Gao G, Grunkemeier GL, Wu Y, Zerr KJ, Bookin SO, et al. Continuous insulin infusion reduces mortality in patients with diabetes undergoing coronary artery bypass grafting. J Thorac Cardiovasc Surg. 2003;125:1007-21.

19. Hruska LA, Smith JM, Hendy MP, Fritz VL, McAdams S. Continuous insulin infusion reduces infectious complications in diabetics following coronary surgery. J Card Surg. 2005;20:403-7.

20. Lazar HL, Chipkin SR, Fitzgerald CA, Bao Y, Cabral H, Apstein CS. Tight glycemic control in diabetic coronary artery bypass graft patients improves perioperative outcomes and decreases recurrent ischemic events. Circulation. 2004;109:1497-502.

21. Mangram AJ, Horan TC, Pearson ML, Silver LC, Jarvis WR. Guideline for prevention of surgical site infection, 1999. Centers for Disease Control and Prevention (CDC) Hospital Infection Control Practices Advisory Committee. Am J Infect Control. 1999;27:97-132.

22. Bolon MK, Morlote M, Weber SG, Koplan B, Carmeli Y, Wright SB. Glycopeptides are no more effective than beta-lactam agents for prevention of surgical site infection after cardiac surgery: a meta-analysis. Clin Infect Dis. 2004;38:1357-63.

23. Finkelstein R, Rabino G, Mashiah T, Bar-El Y, Adler Z, Kertzman V, et al. Vancomycin versus cefazolin prophylaxis for cardiac surgery in the setting of a high prevalence of methicillin-resistant staphylococcal infections. J Thorac Cardiovasc Surg. 2002;123:326-32.

24. Caffarelli AD, Holden JP, Baron EJ, Lemmens HJ, D'Souza H, Yau V, et al. Plasma cefazolin levels during cardiovascular surgery: effects of cardiopulmonary bypass and profound hypothermic circulatory arrest. J Thorac Cardiovasc Surg. 2006;131:1338-43.

25. Berger PB, Velianou JL, Aslanidou Vlachos H, Feit F, Jacobs AK, Faxon DP, et al. Survival following coronary angioplasty versus coronary artery bypass surgery in anatomic subsets in which coronary artery bypass surgery improves survival compared with medical therapy. Results from the Bypass Angioplasty Revascularization Investigation (BARI). J Am Coll Cardiol. 2001;38:1440-9.

26. Serruys PW, Unger F, Sousa JE, Jatene A, Bonnier HJ, Schonberger JP, et al. Comparison of coronary-artery bypass surgery and stenting for the treatment of multivessel disease. N Engl J Med. 2001;344: 1117-24.

27. Zhang Z, Mahoney EM, Stables RH, Booth J, Nugara F, Spertus JA, et al. Disease-specific health status after stent-assisted percutaneous coronary intervention and coronary artery bypass surgery: one-year results from the Stent or Surgery trial. Circulation. 2003;108:1694700 . 